Meta

Journal des traducteurs

Translators' Journal

\title{
La définition des termes scientifiques
}

\section{Adrien Hermans}

Volume 34, numéro 3, septembre 1989

1. Actes du Colloque Les terminologies spécialisées : Approches quantitative et logico-sémantique et 2 . Actes du Colloque Terminologie et Industries de la langue

URI : https://id.erudit.org/iderudit/003064ar

DOI : https://doi.org/10.7202/003064ar

Aller au sommaire du numéro

Éditeur(s)

Les Presses de l'Université de Montréal

ISSN

0026-0452 (imprimé)

1492-1421 (numérique)

Découvrir la revue

Citer cet article

Hermans, A. (1989). La définition des termes scientifiques. Meta, 34(3), 529-532.

https://doi.org/10.7202/003064ar d'utilisation que vous pouvez consulter en ligne.

https://apropos.erudit.org/fr/usagers/politique-dutilisation/ 


\section{LA DÉFINITION DES TERMES SCIENTIFIQUES}

A. HERMANS

Centre de terminologie

Bruxelles, Belgique

\section{LES DEUX COMPOSANTES DU VOCABULAIRE SCIENTIFIQUE}

La différence principale entre la lexicographie et la terminographie, du point de vue linguistique, réside dans le type d'analyse sémantique que ces deux disciplines appliquent au mot ou au terme. La lexicographie part du signe pour aller vers la détermination du concept et relève donc de la sémasiologie. La terminographie part de la notion et recherche les termes qui lui correspondent. C'est une approche onomasiologique.

L'approche onomasiologique est utile dans la mesure où le système conceptuel est stable et cohérent ${ }^{1}$ et où les notions ont trait à des objets, au sens large. Elle s'applique donc bien aux termes techniques. Mais les termes techniques ne constituent qu'une partie du vocabulaire scientifique. Dans leur démarche explicative, les scientifiques utilisent des concepts théoriques, qui ne peuvent pas être traités par la méthode onomasiologique.

Parmi les nombreuses définitions de «terme» 2 , nous retiendrons celle proposée par KOCOUREK (1982: 77) : «Le terme est une unité lexicale définie dans les textes de spécialité». ${ }^{3}$ Un terme scientifique est alors une unité lexicale définie dans un texte scientifique. Toute unité lexicale d'un texte scientifique est donc appelée «terme», à la seule condition d'être définie dans le discours scientifique. Suivant en cela KOCOUREK, nous écarterons dans ce qui suit tout élément normatif (le terme doit être monosémique, univoque, non connoté, prescriptif) de la définition du terme.

On peut distinguer deux types de termes dans le vocabulaire scientifique: les termes techniques et les termes «pour des concepts».

Les termes techniques désignent des observations, des mesures, des expériences, des instruments, etc. Ces objets sont, logiquement, préexistants aux termes. Pour constituer la terminologie d'un domaine technique, on part de ces objets, de leurs notions, auxquelles on assigne des termes, correspondant au découpage du champ notionnel. Le terme n'est pas défini à partir de son fonctionnement dans le discours, de sa signification ou dans sa polysémie éventuellle, mais comme correspondant à une notion particulière dans le champ notionnel.

Les «termes pour des concepts» ou termes théoriques ne se prêtent pas à pareil traitement et cela, pour des raisons aussi bien logiques ou épistémologiques que pragmatiques.

\section{LES CARACTÉRISTIQUES LOGIQUES DES TERMES THÉORIQUES DANS LES SCIENCES}

a. Les termes théoriques ne renvoient pas à des notions préexistantes, à des représentations mentales d'objets concrets ou abstraits. Ils sont par contre utilisés avec une ou 
plusieurs significations. Leur signification dépend de leur fonctionnement dans le contexte. Un terme théorique doit être étudié et défini dans son environnement et selon les paradigmes dans lesquels il figure. Sa signification dépend des rapports qu'il entretient avec les autres termes de l'énoncé, qui le délimitent, et des relations privilégiées entre certains termes dans un énoncé ou une discipline donnée. Un concept est donc tout simplement la signification d'un terme ${ }^{4}$. Il n'est donc pas possible de construire par exemple un arbre notionnel de ces concepts pour structurer préalablement un domaine. La définition de termes théoriques consistera donc avant tout dans la spécification des différentes significations que peuvent avoir ces termes (spécifications chronologiques, par école de pensée, par substrat théorique dans lequel le terme apparaît), de leur valeur heuristique, de leur caractère métaphorique éventuel, de leurs connotations, de leur polysémie et de l'économie linguistique et scientifique que leur confèrent ces caractéristiques.

La signification des termes scientifique est continuellement générée par l'usage que font les scientifiques de ces termes et par la manière dont cet usage est compris par les autres. La signification est «a context controlled process» ${ }^{5}$. Cette imprécision ne provient pas d'une incapacité d'être précis, mais se fonde dans la démarche scientifique même. Les sciences sont des systèmes complexes, qui n'essaient pas d'arriver à une stabilité maximale, laquelle équivaudrait à une stagnation totale. Elles cherchent par contre une instabilité proche de l'équilibre entre détermination et indétermination. Si les concepts scientifiques sont trop déterminés, ils ne fonctionnent plus comme instruments de découverte ou d'explication. Ils restent au niveau descriptif des phénomènes. Si ces termes sont définis avec trop de précision, la définition ne reflète plus leur sens véritable. La précision est obtenue en sacrifiạnt leur signification. C'est en effet grâce à leur caractère métaphorique et connoté que ces termes théoriques fonctionnent comme éléments heuristiques et explicatifs dans l'activité scientifique. Les épistémologues actuels affirment que toute. science se fonde sur une opération de métaphorisation, où les glissements de sens, les analogies et l'ambiguité des concepts de base fournissent les hypothèses et guident l'observation' 6 .

La signification des termes scientifiques n'existe donc pas en dehors de l'usage que font les scientifiques de ces termes, en dehors des procédures communicatives.

Les termes théoriques font en outre partie d'un paradigme. Dans la mesure où une science est constituée d'un ensemble de modèles compétitifs, plusieurs significations d'un terme coexistent dans une même discipline scientifique et même une prolifération de significations ne semble pas gêner les scientifiques, qui n'ont souvent pas besoin de conventions terminologiques.

\section{APPROCHE PRAGMATIQUE}

Les définitions des termes théoriques sont parfois utilisées comme moyen d'argumentation scientifique, mais leur fonction principale est de clarifier la ou les signification(s) de ces termes. Elles ont en général un but didactique. «How to teach another person the meaning of a word ${ }^{7}$.» Il faut donc d'abord savoir pour qui et dans quelle situation une définition peut être utile, avec quelle question et dans quelle situation le lecteur ou l'utilisateur d'un dictionnaire fait appel à une définition. Fait-on appel à une définition dans une situation de lecture (passive) ou d'écriture (active), pour connaître la signification d'un terme ou pour trouver un synonyme, etc. ${ }^{8}$ ? Les types de définitions doivent répondre aux situations d'utilisation des définitions.

Les définitions ne sont généralement pas destinées aux experts. On trouve des définitions dans des manuels et des cours, mais c'est surtout à travers le contexte dans lequel les termes sont utilisés et non à partir de définitions précises que l'étudiant qui s'initie à 
un domaine scientifique doit apprendre la signification de ces termes. On devient expert au moment où l'on connaît l'utilisation admise des termes.

Les définitions sont donc surtout destinées à un public intéressé, non initié, à des scientifiques d'autres disciplines, des techniciens, à un public plus large. Puisque les termes théoriques ne renvoient pas à des choses, celui qui cherche une définition ne veut pas savoir ce que désigne le terme (ce qu'est «x» réellement?), mais il veut pouvoir utiliser le terme d'une manière adéquate, le comprendre dans le sens où il est utilisé par ceux qui sont compétents dans le domaine.

\section{CONCLUSION}

Les définitions des termes scientifiques doivent alors répondre à deux exigences : elles reflèteront les caractéristiques des concepts scientifiques et rendront des connaissances scientifiques accessibles aux non-initiés.

Pour bien rendre compte des caractéristiques de ces termes, la définition donnera leurs conditions d'émergence et de fonctionnement, la spécification du ou des paradigmes à l'intérieur desquels ils fonctionnent, les contextes dans lesquels ils sont utilisés, les rapports qu'ils entretiennent avec d'autres termes. Il n'est pas toujours possible de donner toutes les spécifications ou toutes les significations existantes. Dès lors, une définition ne doit pas être trop précise mais elle doit refléter le consensus le plus large possible parmi ceux qui sont compétents dans le domaine.

Du point de vue de son utilité, une définition doit être lisible et compréhensible. Une définition doit surtout donner les informations utiles pour ceux à qui elle est destinée. Une surabondance d'informations et une trop grande précision sont à éviter.

Souvent, les définitions faites par des experts sont trop liées au paradigme bien précis à partir duquel ces scientifiques construisent leurs propres théories et leurs modèles et ne répondent pas aux règles de formulation établies par la lexicographie et la logique. Le travail de définition doit être confié à un terminologue, procèdera aux opérations suivantes ${ }^{9}$ :

- inventaire des différentes significations du terme et étude de l'évolution de leurs significations;

- décisions concernant le nombre de spécifications contextuelles que l'on peut raisonnablement et utilement introduire dans le dictionnaire ou le vocabulaire ;

- élaboration d'une première définition;

- recherche de la compatibilité de cette définition dans des contextes différents, dans des paradigmes différents, par rapport à d'autres défínitions du même terme, etc. ;

- adaptation éventuelle de la définition;

- spécification des caractéristiques et des besoins des utilisateurs potentiels;

- adaptation éventuelle de la définition en fonction de ces besoins.

\section{RÉFÉRENCES}

1. REY, A. (1977): Le lexique : images et modèles. Du dictionnaire à la lexicologie, Paris, Armand Colin, p. 16.

2. R. Kocourek rappelle les sept définitions les plus courantes dans La langue française de la technique et de la science, Paris, La documentation française, 1982. On trouve aussi dáutres encore dans H. Felber (1984): Terminology Manual, Infoterm, p. 168.

3. R. Kocourek, o.c., p. 77.

4. Le terme «concept» est utilisé par les épistémologues de deux manières différentes. Souvent, ce terme désigne à la fois la dénomination (le terme) et le concept (la signification). Terme et concept sont alors équivalents. D'autres font une distinction entre terme et concept. Souvent aussi on constate un glisssement de sens dans un même texte, glissement auquel le lecteur s'adapte automatiquement. On peut considérer ce processus comme une forme d'économie du langage scientifique : un même terme peut avoir des significations différentes selon le contexte communicatif et sémantique. 
5. DE BEAUGRANDE, R. «Systemic versus contextual aspects of terminology», Terminology and Kwoledge Engineering, Frankfurt, Index Verlag, pp. 7-24.

6. MARCHAL, P. (1980): «Discours scientifique et déplacement métaphorique», La métaphore, Approche pluridisciplinaire, Bruxelles, Faculté universitaires Saint Louis.

7. ROBINSON, R. (1972): Definition, Oxford, Clarendon Press, p. 93.

8. WIEGAND, H. E. (1985): «Einige grundlegende semantische-pragmatische Aspekte von Wörterbucheintragen, L. Zgusta (1985): Probleme des Wörterbuchs, Darmstadt, Wissenschaftliche Buchgesellschaft, pp. 343-377.

9. DE GROOT, A. D., F. L. Medendorp (1986): Term, Begrip, Theorie. Inleiding tot de signifische begripsanalyse, Amsterdam, Boom Meppel. 